\begin{tabular}{|c|c|}
\hline \multicolumn{2}{|c|}{ SGML AUTHORING TOOLS } \\
\hline ADEPT Publisher & ArborText \\
\hline Author for Word & Microsoft \\
\hline Cyberleaf & Interleaf \\
\hline HotMetal & SoftQuad \\
\hline Intellitag & WordPerfect \\
\hline \multicolumn{2}{|l|}{ Quaterdeck } \\
\hline SGML Editor & Grif \\
\hline Tag Wizzard for Word & NICE Technologies \\
\hline \multicolumn{2}{|c|}{ ON-LINE DOCUMENT DELIVERY SYSTEMS } \\
\hline RightPages & AT\&T/Springer \\
\hline GUIDON & OCLC \\
\hline WEBZ & OCLC \\
\hline$w W W$ & CERN... \\
\hline Hyper-G & Graz University \\
\hline \multicolumn{2}{|c|}{ PORTABLE DOCUMENT SOFTWARE } \\
\hline Acrobat & Adobe \\
\hline Common Ground & No Hands Software \\
\hline Replica & Fallon \\
\hline Envoy & WordPerfect \\
\hline
\end{tabular}

lel" and the "perpendicular" scenarios (one could say evolutionary and revolutionary).

In the parallel scenario, the electronic journal only exists in parallel with the usual paper version, as will be the case for Physics Review Letters from this July. This has advantages in terms of shortening communication delays (the electronic form generally appears before the paper version), automatic searching for information, etc. The storage problems of libraries call for the extensive use of CD-ROMs for archival, and the access to information by scientists in underdeveloped countries may become easier.

In the perpendicular scenario, the traditional journals disappear, or become so different that their new wave function has a very little overlap with what is was before (hence the term). For instance, an individual laboratory or a group of laboratories may set up servers with their own rules for ensuring scientific quality, safety of archival, etc. Our imagination is in fact the only limit because the nature of present journals does not have to be transposed. Take scientific evaluation which remains an absolute necessity. One can conceive of systems of databases with different levels - those where all articles are accepted and remain forever, those for articles after they have been selected by qualified readers, etc.

We have to be ready to cope with and analyse numerous possibilities. This is the main challenge today, and in addressing it the workshop reviewed the state-of-the art and the various approaches before discussing how, as physicists, we should act in order to be reasonably prepared whatever the outcome. The problem is not only to develop better software for authoring and viewing, but also to address legal issues such as copyright. Perhaps the most important aspects concern efforts to harmonize developments across national borders and scientific disciplines.

\title{
Scientific Publishing without Publishers
}

\author{
Jean Zinn-Justin argued at the EPS workshop Electronic Publishing in Physics \\ (Paris, 2-3 March 1995) that electronic documents will allow essential changes to \\ be made in the way physicists publish articles.
}

It is becoming increasingly obvious that electronic publishing is not paper publishing with electronic means. The combination of electronic production of scientific documents, $T_{E} X$, electronic mail, Internet and now the Web, and the tremendous success of Los Alamos-type preprint database servers, is resulting in a true revolution. The whole publishing process has therefore to be re-examined.

As there are many types of publications playing a variety of different roles, in discussing the implications of electronic publishing it is necessary to focus on certain types. Scientific contributions for which authors expect to be paid, and reviews and books for which the paper form in bound volumes is especially useful, will remain in the near future in the hands of traditional publishers.

In focussing on scientific articles and journals one needs to analyze the role they play in general scientific activity, and to better understand their properties and the new possibilities. Since the electronic revolution will directly affect traditional publishers, it raises a natural question: will the future see scientific publishing without publishers? I shall argue, in particular, that electronic publishing gives us an opportunity to survive the slow collapse of the refereeing system - one of the principle cornerstones of paper publication.

\section{Crisis in the Referee System}

Although evaluation and selection are among the most critical items in publishing an article, publishers also undertake to: - Gather scientific information, select (with the help of scientists) contributions worth publishing, and organize a discussion between referees and authors, which sometimes helps improve the content. Note that the paper medium imposes an artificial coupling of two different tasks, namely immediate evaluation of a piece of work and a

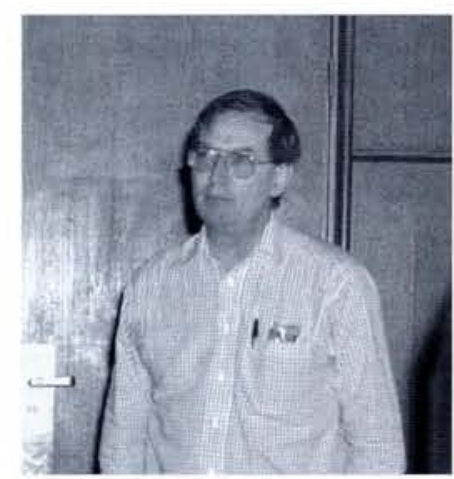

means for scientists to communicate the results of their research.

- Edit and format articles.

- Disseminate and archive information.

- Help organize information, although often only in minor ways (by creating topical letters, comment sections, indexes, etc.).

A few empirical remarks are appropriate.

\section{Increased productivity}

The number of published pages is increasing exponentially, but alas not the scientific content, or at least not at the same rate. I can only guess the reasons: increase in the number of physicists; increase in the physicists' "productivity" owing to external pressure (the feeling that a healthy number of published papers is essential for a scientific career); improved production tools (e.g., computers).

Referees are no longer able to control the flood. This is obvious to physicists who have been in the editorial business for some time (those who are not have generally stopped reading journals anyway). Referees receive too many papers which are poorly written and highly specialized. Assessing relevance and novelty therefore becomes an almost impossible task. Moreover, the less interesting papers are the more demanding in terms of the referee's time. If a referee becomes too selective the author generally fights back, asks for another referee, then goes to another journal. The author is really unlucky if a paper is not published in the end, and several referees eventually discover that their time has been totally wasted. Finally, if existing journals become too selective, new journals are created. Moreover, in a world in which the success of a journal is measured by the number of published pages, it cannot be expected that publishers themselves will try to discourage authors.

\section{Shortcomings}

Paper publication has two serious shortcomings. While a conscientious referee may have written a long and argued report, the final outcome can only be "yes" or "no", even when the report would prefer "maybe". Moreover the answer "no" means depriving, perhaps on subjective grounds, a physicist

Jean Zinn-Justin (zinn@amoco.saclay.cea.fr), seen here speaking at the EPS Electronic Publishing in Physics workshop, is a theoretical physicist with CEA Saclay. He has served as the Editor-in-Chief of $J$. de Physique and is currently Honorary Editor of J. Phys. A, Associate Editor of Nucl. Phys. B, Editor of Fortschritt der Physik, and Co-editor of Proc. Les Houches Summer Schools. 


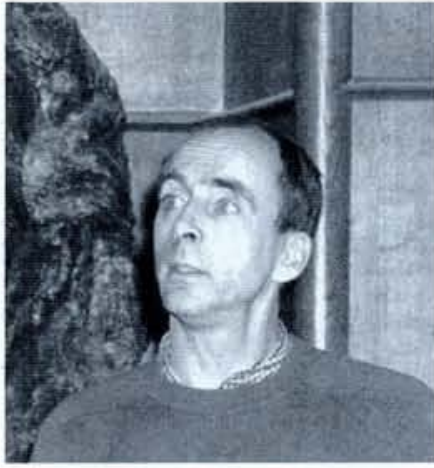

B. von Sydow describing the EUROMATH project that aims to create a SGML-based authoring environment for mathematics.

of the possibility to add a paper to a list of publications. The preprint system, on the other hand, has for many years provided an alternative means of communicating scientific information, at least in most established laboratories.

As the only possible evaluation for paper publication is based on acceptance or rejection, evaluation remains unique, at least if a paper is accepted: it discriminates poorly even if referees do a serious job and discuss the paper at length. Once a paper is published, its content can only be discussed through another publication. Errata published months later, and which nobody reads, are the only way to point out errors. All this in turn contributes to the dilution of scientific information.

To answer the comment that established journals reject a certain fraction of submitted papers (e.g., $30 \%$ in high-energy physics), it should noted that this fraction has remained approximately constant while the number of papers has increased dramatically. Refereeing therefore, while not being totally irrelevant, is simply becoming increasingly ineffective in science.

In conclusion, we produce at increasing cost information of decreasing value where the rate of dilution of scientific information has become excessive. Using the traditional production system, nothing can really be done to prevent further deterioration. Drastic changes are required so that we can use research funds in a more useful and efficient manner.

\section{The Electronic Route}

The question of electronic publication does not reduce to a discussion of the new tools which could facilitate the production and circulation of scientific documents. Electronic articles, owing to their "plasticity" and speed of transmission, have entirely new properties which force one to rethink the entire publishing process. In reorganizing publication we have to keep in mind that the only goal is to better satisfy the different needs of the scientific community. In particular, there is absolutely no justification for reproducing the shortcomings of paper publication in the electronic form.

For example, the Letter concept emerged when papers became too numerous as a means to speed up the dissemination of important results. The Letter format is a constraint typically required by the paper form. It all too often leads to incomprehensible articles generated by taking a longer article and randomly eliminating sentences to sa- tisfy the length criterion. Straightforward extrapolation of established results have a better chance to be published than really novel contributions because some important new results require more explanation.

These shortcomings can be eliminated in electronic publication since length is no longer related to the speed of distribution. If we want to emphasize some articles, this should only be done according to scientific value, which is enhanced by improving readability.

It is clear, of course, that much of the manipulation of typescript can be, and therefore should be, done electronically. However, one must first define the tasks which remain or become necessary in the electronic age. Returning to the items listed at the beginning:

Collect scientific information. This is already done in a fairly automatic way in Los Alamos-style databases, where it is rapid and inexpensive. We do not need to worry about a lack of storage space since storage media increase continuously in capacity and decrease in price (the cost per gigabyte stored has decreased by about a factor of four in five years, a rate scientists will not be able to match, at least with standard documents). One should note, however, that it is essential to have all papers in a field gathered in a unique database (logically unique, of course) for easy access and searching.

Selection is no longer necessary, or even useful, because there is absolutely no reason to deny a physicist the right to communicate research results. The dissemination of raw, scientific results by sending them to a database is decoupled from scientific evaluation. However, we may want to restrict the database to what we call physics; this could require some form of non-automated intervention.

Formatting and editing aims to ensure scientific accuracy and readability. Whether reasonable standards have been achieved is for the scientific community to decide. With the appearance of $T_{E} X$ and the widespread availability of computers and printers, formatting and editing is now routinely performed by authors directly, with or without local help. I believe that authors after a few years usually reach a satisfactory level so external intervention on typescripts merely endangers scientific accuracy while hardly improving readability. So these two tasks should now in general be left to authors.

Dissemination of scientific information. This task is now performed in a much faster, cheaper way by electronic means. Information is easier to retrieve, and even to recover in paper form, at least when there exists a Postscript version. One should compare printing on your local printer, which immediately provides you with a neat copy of a paper, with struggling in the library to photo- copy a bound volume of articles.

Archiving in its simplest form is solved using trivial means, although one has to worry about having enough mirrored electronic copies and ensuring that files are mounted using the latest storage medium wherever possible.

Organization and structuring of scientific information for retrieval purposes will become increasingly important, but it is hardly treated by publishers at present. One needs to develop ways to transform a store of raw scientific information into a real database, for we have learnt that having too much unstructured information available is close to having no information at all. This task contains a technical component involving the development of software to browse documents. An example is provided by hypertext capabilities, which in their most sophisticated forms imply artificial intelligence and the advanced techniques of computer science. However, it is essential to take into account the importance of the large quantity of $T_{E} X$ articles which already exists and the proven flexibility of $T_{E} X$ for processing mathematical texts. A solution which requires physicists to suddenly write their articles in some new text programming language is doomed to fail.

\section{Dynamic Evaluation}

To structure information it is necessary to evaluate it, which requires experts. The present system based on paper publication has reached, and probably passed, its limits. It has to be overhauled, something that can be achieved using electronic documents. The optimum strategy is not clear yet, and some experimentation will be required before we find a new, stable mode of operation. Physicists are working on the problem and the first implementations should appear shortly.

The principle observation is that the evaluation of electronic articles will be done dynamically so important documents will, in a certain sense, remain alive. But a problem has first to be solved, namely when does an article take its final form? This question is essential for subsequent referencing, for once an article is finalized the evaluation process can begin.

In a first stage at least, evaluation can occur spontaneously as a by-product of commenting. With the author's authorization, it will be possible to append signed and dated comments to articles (the author will also be allowed to add remarks or corrections). Alternatively, some colleagues will be urged to make public their personal appreciations. It is expected that physicists will be more inclined to comment on articles they enjoy reading than on those of marginal interest they only read, under pressure, as referees. It is also believed that this new type of evaluation will be more useful to the community. 
The form of comments of course raises many questions: Which forms will be accepted? Who decides that a comment is suitable? Will some action be taken to resolve conflicting opinions? Will spontaneous refereeing be sufficient, or will it still be necessary to solicit evaluation, as in the traditional system? The evaluation, to be useful, has to be easily accessible, and this affects both the precise form of the evaluation, and the electronic tools needed to retrieve it (can one think here in terms of a Michelin guide for articles in say quantum gravity?).

It is often said that in the traditional system, interaction between referees and authors leads to improved articles whereas in the new system this interaction will disappear. First, from my own editorial experience, this improvement effect should not, unfortunately, be overestimated. Moreover, since authors will slowly discover that it is useless to accumulate unreadable (and therefore unread) articles, they will be subject to a renewed pressure to write better articles, an effect which in the long run may more than offset the temporary inconvenience of having no interaction.

Secondly, the logic of the new system requires comments to be signed, leading to the disappearance of the review system based on anonymous referees which has some merit. Whether this will have any significant impact is unclear. But whatever the ultimate form of the evaluation and commenting process, I believe the electronic medium opens up new and exciting possibilities which will eventually be of tremendous importance for the scientific community.

\section{ACKNOWLEDGEMENT}

Although it does not necessarily reflects all his views, this contribution has benefitted from many interesting discussions with Paul Ginsparg from the Los Alamos National Laboratory, USA.

\section{Reflecting an Enlarged Community}

\section{EUROPEAN JOURNAL OF PHYSICS}

\section{Samuelsson (Linköping University) took over late last year from A.B. Pippard as the Editor of the European Journal of Physics.}

The European Journal of Physics (EJP) is a relatively new journal which publishes articles destined for university-level lecturers. So articles must be neither too long nor too theoretical. However, it has been found that many university staff are unaware of the existence of this very useful journal. It is therefore important to publicise the journal more widely, and every effort will be made to do this. It should also be possible to make the journal more attractive by introducing new subjects, notably in experimental physics. But this needs to be discussed first by the Editorial Board, whose number will be brought up to the full complement of 15 by new appointments made by EPS Executive Committee. Proposals for the Board members now come the new EPS Interdivisional Group for Physics Education which members of all national societies can join. This reflects the importance of physics education

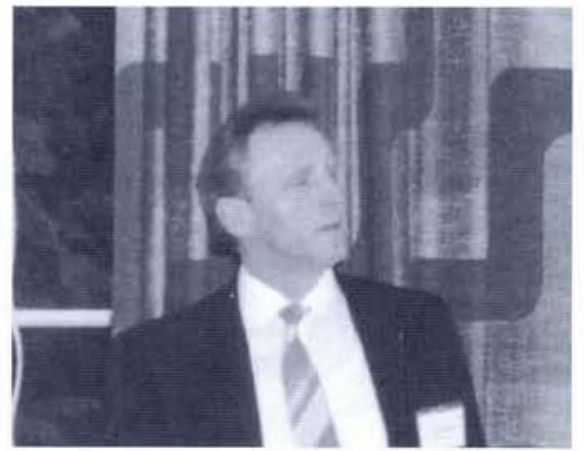

L.Samuelsson, the Editor of the European Journal of Physics, speaking at the 1995 EPS Council.

in EPS - a feature that EJP will definitely aim to highlight.

\section{EUROPHYSICS LETTERS}

R. Balian handed over the position of Editor-in Chief of Europhysics Letters to F.A. Gianturco (Chemistry Department, University of Rome) on 1 April.

I must first thank the European Physical Society for having allotted me a responsibility which has been extremely rewarding. My task as Editor-in-Chief of Europhysics Letters (EPL) has certainly been easier than that of my predecessors, N. Kurti and W. Buckel. It was they who had to set up a new journal - actually the only scientific journal (together with Astronomy \& Astrophysics) which is published under the simultaneous auspices of a large group of national societies. It was a pleasure for me to continue their work and to help in developing an enterprise which is exemplary in the construction of a "Europe of Science". Since its creation in 1986, EPL has reached a steady position: the number of subscribers is $\approx 900$, and the journal publishes $\approx 450$ articles p.a.
The quality of our journal relies mainly on its Co-editors. Their competence and devotion are certainly major elements of its success, and I wish here to express especially the gratitude of our community to them. One of their main difficulties is to coordinate the work of the referees. They have succeeded these last years in shortening the delay to acceptance, which now has a median of eight weeks for manuscripts that do not need revision (16 weeks with revision). This is still too long, but our rejection rate $(41 \%$ of the submitted manuscripts in 1994 - see table) and our revision rate $(80 \%$ of the accepted Letters) indicate the value of the work of the referees and Co-editors.

It has also been a pleasure for me to work with our efficient editorial office, and the technical teams should also be praised. Considering that EPL is edited in Geneva, composed in Bologna, printed in the west of France, and dispatched from near Paris, the present delay of only 5 to 6 weeks between the acceptance of an article and its publication is a remarkable achievement of our European cooperation.

As regards our Management Board, I shall give only one, very recent, example of the importance of its contribution. It has recently been decided that, from next July on, the titles and abstracts of all Letters will be available free-of-charge on World-Wide Web (http://www.edphy.fr/epl) as soon as they are accepted. This step, which should be appreciated by all physicists, reflects a permanent concern for modernization.

Although the situation thus looks rather satisfactory, the collective effort of our community should be used to remedy two distortions. First, the geographic distributions of authors submitting manuscripts and of subscribers does not yet fully reflect the relative importance of physics in various countries. This originates, of course, from some ancient traditions; but we feel that, at a time that the EPS is enlarging its audience, physicists from all over Europe should regard $E P L$, the journal run by our Society, as their privileged means for communicating new ideas and results in the form of Letters.

Second, more than one-half of the articles that we publish are somehow related to condensed matter or to statistical physics. Here again, historical reasons explain the distortion - a distortion which has some advantages. Nevertheless, the vocation of EPL is more universal since it is intended to include important and novel results which not only satisfy the specialist but are also of interest to a rather broad community. Research is often threatened by overspecialization: everyone is so busy in a particular field and often does not always realize the interest, in the long run, of a wide scientific culture. However, many discoveries were made by transposing an idea from one field to another. With this in mind, EPL is open to physicists from all fields: we urge them to submit manuscripts in domains where our journal is not yet well established, especially 